EPJ Web of Conferences 32, 02005 (2012)

DOI: $10.1051 /$ epjconf/20123202005

(C) Owned by the authors, published by EDP Sciences, 2012

\title{
Real-time control of multiple MHD instabilities on TCV by ECRH/ECCD
}

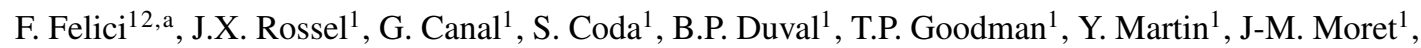
O. Sauter ${ }^{1}$, D. Testa ${ }^{1}$, and the TCV Team ${ }^{1}$

1 Centre de Recherches en Physiques des Plasmas, École Polytechnique Fédérale de Lausanne (EPFL) (CRPP-EPFL), Lausanne Switzerland, CH-1015

2 Present address: Eindhoven University of Technology, Den Dolech 2, P.O. Box 513, Eindhoven, $5600 \mathrm{MB}$, The Netherlands.

\begin{abstract}
Highly localized deposition of ECRH/ECCD is particularly suited for MHD control, in particular when combined with real-time beam orientation and power control capabilities. The powerful (4.5MW) and flexible ( 7 steerable launcher) EC system on TCV has recently been complemented by an equally flexible digital real-time control system with the aim of developing and testing integrated MHD control methods [1]. Sawtooth pacing is one such method [2]. The crash time of stabilized sawteeth can be precisely controled by removing the EC power at a given time after the last sawtooth crash, causing the crash to occur at a short and reproducible time thereafter. This control strategy is combined with efficient neoclassical tearing mode (NTM) preemption by depositing power at the mode rational surfaces only during a short time synchronized with the island-seeding sawtooth crash. If an NTM appears nevertheless, full power is applied to stabilize the mode. The real-time steerable launchers have also been employed to stabilize fully saturated NTMs and to investigate the precise requirements for deposition localization for full island stabilization. Finally, though ELM dynamics is markedly different, recent results show that ELM pacing is possible using a similar control technique as used for sawtooth pacing. In this case, edge EC power is removed after each ELM, and is reapplied after a programmable time interval. The ELM period can be real-time controlled by adjusting the length of this interval. While the overall trend conforms to the increase of ELM frequency with increasing power, this technique provides a means to significantly regularize the ELM cycle.
\end{abstract}

\section{Introduction}

ECRH/ECCD constitutes a prime surgical tool for controlling plasma MHD mode activity. Recent experiments on TCV have focused on developing and testing several new methods of MHD control employing real-time steerable launchers and variable EC power levels. Control of the sawtooth instability, the edge localized mode, and neoclassical tearing mode is thus demonstrated. This paper provides an overview recent experimental results. More detailed information can be found the cited references.

\section{ECRH/ECCD system and control hardware}

The MHD control experiments presented in this paper fully utilise the operational flexibility of the TCV X2 ECH system, which features 6 independent, poloidally steerable launchers injecting power

\footnotetext{
a e-mail: f.felici@tue.nl
} 


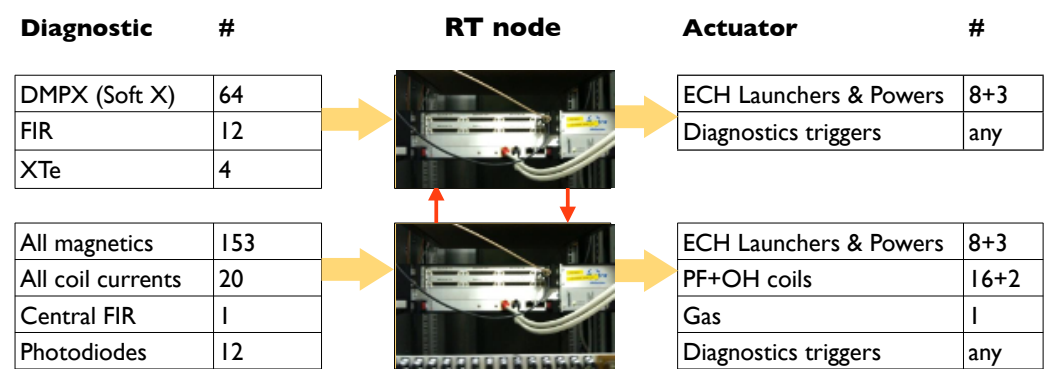

Fig. 1. Schematic representation of control system nodes and connections used for the experiments presented in this paper. Each nodes can run at a different clock rates and communicate with other nodes via a shared memory. Adapted from [5].

from 6 gyrotrons powered by two independent power supplies (3 gyrotrons per power supply [3]). These powerful heating and current drive actuators receive commands from the recently commissioned digital control system [4]. This system processes information from serveral multi-chord diagnostics and provides command references to the poloidal steering mirrors and/or gyrotron power supplies (cf. Figure 1). Several nodes make up the system, and each node can be tasked with a different control algorithm. In typical MHD control experiments, the system operates independently from the main plasma control system which controls bulk parameters of the plasma (current, position, density, etc). In practice, the first node contains a real-time sawtooth detector based on x-ray signals, as well as the real-time decision-logic algorithms to actuate the various phases of the discharge. This node operates at a clock rate of $10 \mathrm{kHz}$ in these experiments. The second node treats signals from magnetics and photodiodes at $50 \mathrm{kHz}$ and runs a digital PLL (Phase Locked Loop) for NTM detection and/or an ELM detector algorithm based on a threshold of the photodiode $\left(\mathrm{H}_{\alpha}\right)$ emissions.

\section{Sawtooth pacing}

The sawtooth instability appears as a sudden relaxation of the core temperature and density profiles. While its period can often be controlled by appropriately changing the deposition location ECCD in the vicinity of the $q=1$ surface [6], the performance of this type of control is often limited by the mechanical characteristics of the launcher. The effect of localized ECCD can be qualitatively understood as accelerating or retarding the time at which a crash threshold (often a condition on the magnetic shear $s_{1}$ at $q=1$ ) is reached. In this case, control by changing the deposition location has its origin in varying the time rate of change of the magnetic shear. Sawtooth pacing [2], instead, relies on removing the stabilizing power at $q=1$ during the sawtooth cycle. This causes the shear to increase more rapidly and the crash threshold to be attained sooner. It is then possible to control the time of each sawtooth crash by varying the time at which the power is switched off ( $\tau_{\text {set }}$ in figure 2$)$.

Using this technique, it is possible to regularly control the sawtooth, as shown in figure 3 , and even to prescribe the period of each individual sawtooth. In sawtooth pacing, each crash is detected by a sawtooth detector based on a discrete-time high-pass filter. A related technique, called sawtooth locking, does not rely on real-time detection of the sawtooth crash time but instead uses fixed modulation of the ECCD power to which the sawtooth can lock in some cases [M. Lauret, this conference and [7]].

\section{ELM pacing}

Recent TCV experiments have also focused on the influence of edge ECH on the Edge Localized Mode (ELM). In H-mode plasmas with type-I ELMs (verified by checking that the ELM frequency indeed increases with power), the ELM frequency appears also to increase as the deposition location comes closer to the edge, even as the total power decreases as the absorbed fraction decreases [8]. This counter-intuitive result suggests that the the ELM dynamics do not only depend on the total power, 


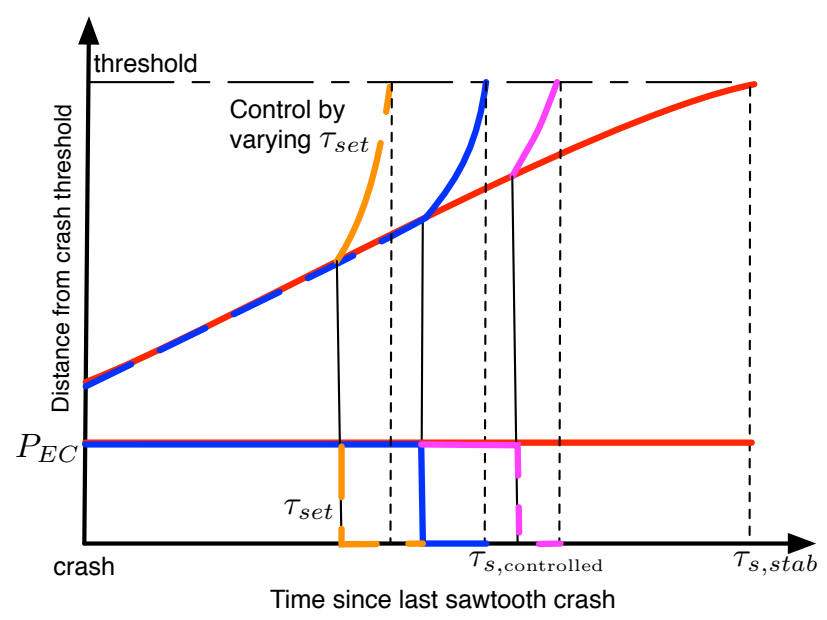

Fig. 2. Cartoon illustration of sawtooth pacing principle: the EC power which otherwise slows the evoltuion of the plasma towards the crash threshold is switched off during the sawtooth cycle, giving precise control of the sawtooth period by varying the switch-off time $\tau_{\text {set }}$ (Figure from [5])
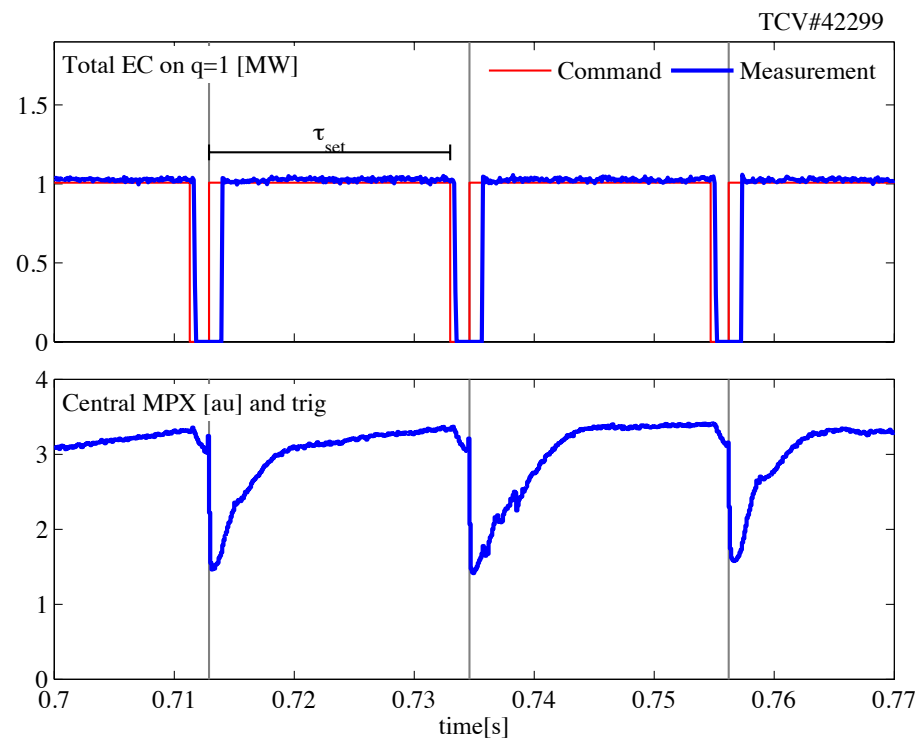

Fig. 3. Demonstration of sawtooth pacing in TCV. The gyrotron power is switched on after each detected crash and switched off after a pre-determined period $\tau_{\text {set }}$. The sawtooth crash is seen to appear shortly thereafter. The EC system hardware causes a short delay between the power command and obtained forward power. From [5].

as is commonly assumed in type-I ELM models, but also on the details of the deposition location. The sensitivity of the ELMs to the edge power suggests that ELMs may be susceptible to pacing like the sawtooth instability. In this case, the EC power destabilizes the ELM, so power should be added in order to stimulate the ELM "crash". Figure 4, illustrates this feature. Indeed, it is possible to regularize the ELMs and prescribe the time of their apprerance using this method, although the operational range is limited between the maximum ELM period (without EC power) and minimum ELM period (with maximum EC power). More detailed results are given in [9] and [5]. 


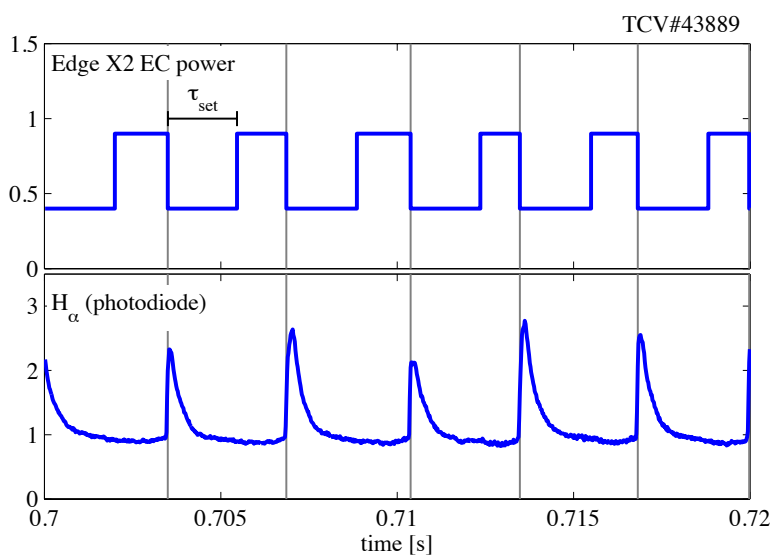

Fig. 4. Detail of ELM pacing: the EC power is switched off after the detection of each ELM crash. The power is switched back ON after a prescribed time, stimulating the appearance of the next ELM. From [5].

\section{NTM control}

NTMs are regularly destabilized in TCV, their origin being either local current profile effects or to seed-island generating sawtooth crashes. Control tools for NTM stabilization with steerable mirrors have recently been tested on TCV. The so-called "scan-and-stop" method is a rather simple NTM control scheme, which however does not require dedicated hardware or complex signal processing and equilibrium reconstruction like many other proposed schemes. This strategy consists of the following steps:

- Pre-determinine the approximate range of location of the rational where then NTM is expected (usually the $q=2$ surface) from equilibrium reconstruction and determinine the corresponding poloidal mirror angle range.

- During the shot, monitor the presence of NTMs in real-time using a digital PLL (phase-lockedloop) which processes a Mirnov probe signal with $50 \mathrm{kHz}$ sampling rate.

- When a mode is detected, scan the launcher angle across the range expected to cross the rational mode surface.

- When the mode is stabilized, or reduced below a given threshold amplitude, hold the mirror position in place.

Two examples of successful NTM stabilization using this method are shown in figure 5. NTMs are stabilized both with an EC beam coming from the outside, and one coming from the inside with respect to the mode. Analyzing the deposition location at the time of stabilization reveals that the deposition locations are within $1 / 2$ beam width of each other. The marginal island size, i.e. the size of the island at the time of full NTM stabilization, also appears to be independent of the direction of the incoming EC beam. For more details and further discussion, see [1].

\section{Integrated control of sawteeth and NTMs}

Usually, large sawteeth are not desirable since they can destabilize NTMs at lower $\beta$ [10]. However, preemptive action can be taken to stabilize the NTM before it grows into a saturated island [11]. Since the crash time is known, one only needs to apply preemptive power for a short time around the instant of the sawtooth crash making the preemptive action more efficient.

Sawtooth pacing and NTM preemption, based on the crash time knowledge, has been combined with NTM suppression to form an integrated MHD control algorithm. In this case, two gyrotrons $(2 \times 500 \mathrm{~kW})$ are aimed to just outside the $q=1$ location. Sawtooth pacing is used to generate longer sawteeth, and 3/2 NTMs are destabilized at sufficiently long sawtooth periods. To counter this, another 

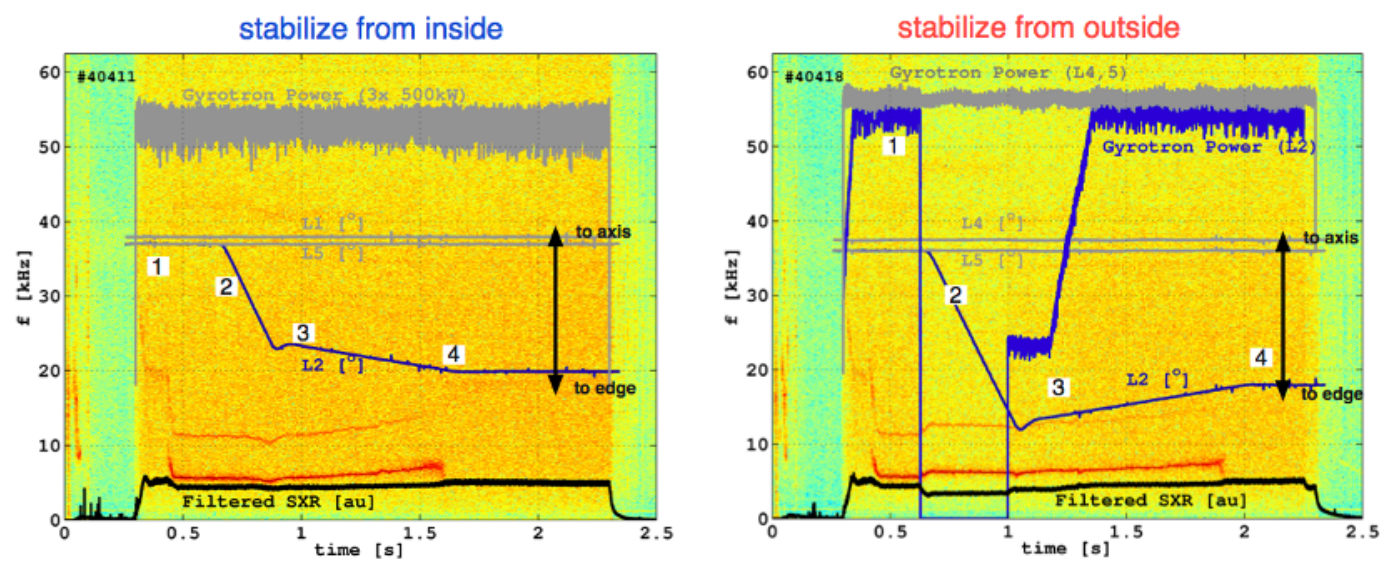

Fig. 5. Stabilization of NTMs by sweeping the ECCD deposition location towards the island from both inside (left) and outside (right). An NTM is first created by three gyrotrons (total 1.5MW) injecting co-ECCD at approximately $\rho_{\psi}=0.3$ (1). Some time after the magnetic island is detected, one of the launchers is moved towards the location of the island (2) and then slowly scanned towards the expected location (3). When the mode disappears, the angle is held constant (4). Note that when stabilizing from the outside (right panel) the gyrotron power is temporarily switched off to allow the launcher angle to go to the requested location without depositing power on the island. From [5].

gyrotron $(500 \mathrm{~kW})$ is aimed at the $q=3 / 2$ surface location. The optimum injection angle for this gyrotron has been decided based on NTM suppression experiments with sweeping launcher angles described in Section 5. This gyrotron is switched on for $7 \mathrm{~ms}$ at the time the power at $q=1$ is removed. This means that the power is present at the time of the seed-island generating sawtooth crash, and is removed during the quiescent phase before the next sawtooth crash.

Figure 6 illustrates that this control strategy is effective. The case without NTM preemption (left) shows NTM activity appearing periodically and then being rapidly suppressed by the NTM suppression scheme. On the other hand, adding $300 \mathrm{~kW}$ of well-timed preemptive power completely prevents NTMs from developing.

\section{Conclusions and Outlook}

This paper has shown new results obtained during TCV experiments on real-time MHD control. Individual sawtooth crashes and ELM events have been shown to be precisely controllable by suitably timed EC power. NTMs in TCV can be stabilized simply by scanning the EC deposition location across the pre-calculated, approximate rational surface location, and stopping once the NTM is stabilized. NTM stabilization is combined with sawtooth pacing in a preemptive NTM suppression strategy, where power is added just at the sawtooth crash time to preempt a possible seed island from growing into a fully saturated NTM. This provides an integrated MHD control method where multiple EC sources are used for stabilizing multiple MHD modes.

The results shown in this paper constitute a step forward in proving tokamak MHD control strategies on the road towards ITER. The strategies implemented here are all compatible with the ITER EC system hardware. In particular, sawtooth and NTM preemption could both be performed by the same upper launcher. The power removed from the $q=1$ surface for sawtooth pacing can be rapidly redirected to the $q=2$ surface for NTM preemption. With typical mirror timescales measured in fractions of seconds [12] and resistive timescales of several seconds this appears feasible, though dedicated simulations should confirm this. These simulations could also address the potential benefits in terms of fusion gain of operating an inductive H-mode with large stabilized sawteeth, providing longer intercrash periods of higher density and temperature. On existing tokamaks, these MHD control strategies 

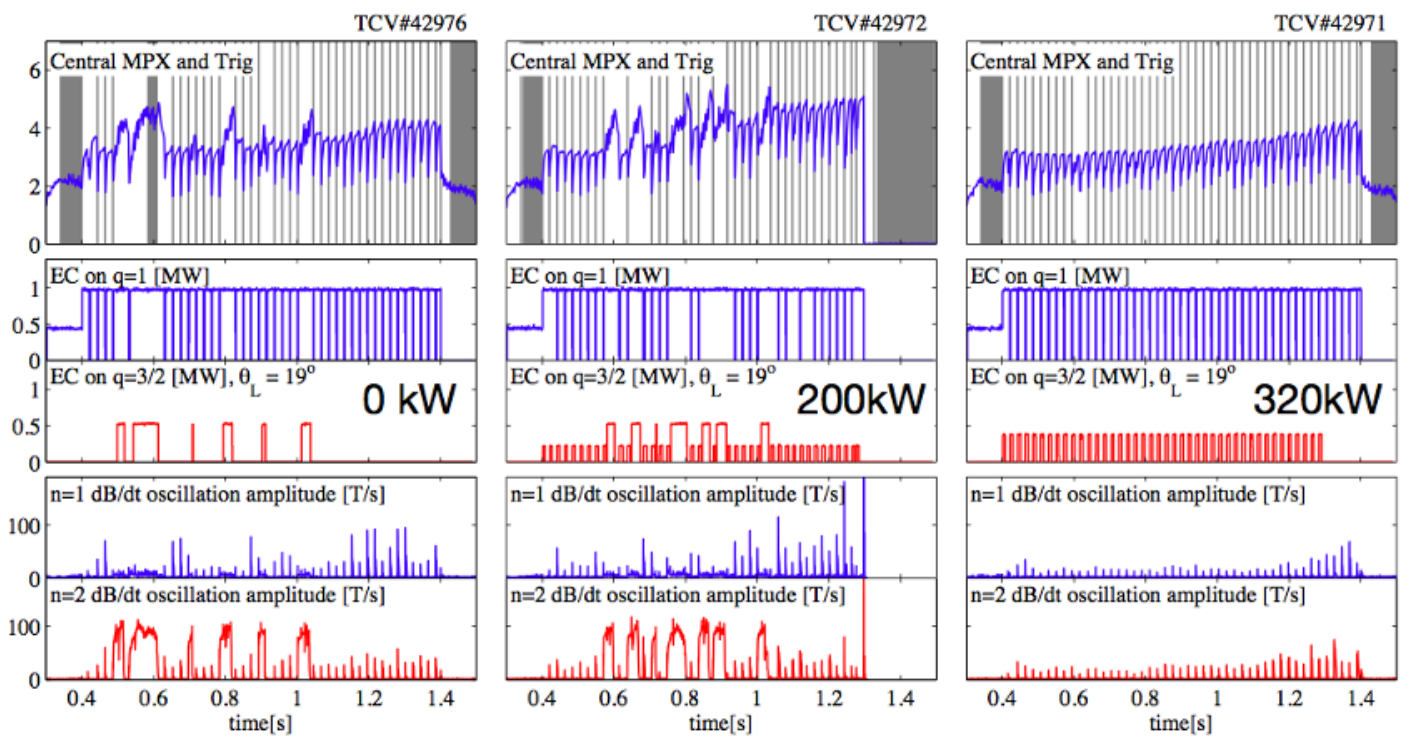

Fig. 6. Preemptive stabilization by $300 \mathrm{~kW}$ of power, periodically applied a the $3 / 2$ surface, successfully prevents NTMs from appearing in this stabilized long-sawtooth plasma. Reproduced with permission from [1].

provide flexible and reliable tools for further physics studies and for enhanced experimental reproducibility. Also, testing these strategies in existing large tokamaks on high- $\beta$ plasmas with significant fast-particle content would provide a further confirmation of their effectiveness. "Whipping the plasma into shape" rather than allowing it to do what it likes may ultimately be the best path to fusion power.

This work has been supported in part by the Swiss National Science Foundation

\section{References}

1. F. Felici, T.P. Goodman, O. Sauter, G. Canal, S. Coda, B. Duval, J. Rossel, the TCV Team, Accepted for publication in Nuclear Fusion (2012)

2. T.P. Goodman, F. Felici, O. Sauter, J.P. Graves, Physical Review Letters 106, 245002 (2011)

3. T.P. Goodman, the TCV team, Nuclear Fusion 48, 054011 (2008)

4. J. Paley, S. Coda, B. Duval, F. Felici, J.M. Moret, Architecture and commissioning of the TCV distributed feedback control system, in 17th IEEE-NPSS Real Time Conference (RT) (2010), pp. 1 $-6$

5. F. Felici, Ph.D. thesis, Ecole polytechnique fédérale de Lausanne EPFL, Lausanne (2011)

6. J. Paley, F. Felici, J. Berrino, S. Coda, N. Cruz, B. Duval, T. Goodman, Y. Martin, J.M. Moret, F. Piras et al., Real Time Control of Plasmas and ECRH Systems on TCV, in Proceedings of the 22nd IAEA Fusion Energy Conference (IAEA, Vienna, 2008), pp. EX/P6-16

7. M. Lauret, F. Felici, G. Witvoet, T. Goodman, G. Vandersteen, O. Sauter, M. de Baar, the TCV team, Nuclear Fusion 52, 062002 (2012)

8. J. Rossel, J.M. Moret, S. Coda, et al., Nuclear Fusion 52, 032004 (2012)

9. J. Rossel, Ph.D. thesis, Lausanne (2012), http://library .epfl . ch/theses/?nr=5311

10. I.T. Chapman, R.J. Buttery, S. Coda et al., Nuclear Fusion 50, 102001 (2010)

11. O. Sauter, M.A. Henderson, G. Ramponi, H. Zohm, C. Zucca, Plasma Physics and Controlled Fusion 52, 025002 (2010)

12. A. Collazos, R. Bertizzolo, R. Chavan, F. Dolizy, F. Felici, T. Goodman, M. Henderson, J.D. Landis, F. Sanchez, Plasma Science, IEEE Transactions on 38, 441 (2010) 